\title{
Einführung des «European Exam in General Cardiology»
}

\section{Felix C. Tanner ${ }^{\mathrm{a}}$, Michael J. Zellweger}

a Prof. Dr. med., Präsident der Weiterbildungs- und Prüfungskommission der Schweizerischen Gesellschaft für Kardiologie (SGK)

${ }^{b}$ Prof. Dr. med., Präsident der Schweizerischen Gesellschaft für Kardiologie (SGK)

Aus Gründen der besseren Lesbarkeit wird auf die gleichzeitige Verwendung männlicher und weiblicher Sprachformen verzichtet. Sämtliche Personenbezeichnungen gelten gleichwohl für beiderlei Geschlecht.

Der Vorstand der Schweizerischen Gesellschaft für Kardiologie (SGK) hat im letzten Jahr beschlossen, den schriftlichen Teil der Facharztprüfung Kardiologie zu erneuern. Dementsprechend wurde das Weiterbildungsprogramm Kardiologie angepasst; letzteres ist abrufbar unter http://www.fmh.ch/bildung-siwf/fachgebiete/ facharzttitel-und-schwerpunkte/kardiologie.html.

Die Erneuerung der schriftlichen Facharztprüfung macht aus folgenden Gründen Sinn:

1. Die Multiple-Choice-Fragen, die aus mehreren Lehrmitteln generiert wurden, genügen den Anforderungen für eine Prüfung nicht mehr, weil der Fragenpool zu klein war, zuwenig häufig angepasst und im Hinblick auf die Eignung nicht systematisch analysiert wurde.

2. Das European Exam in General Cardiology (EEGC) wurde von der European Society of Cardiology (ESC) in Zusammenarbeit mit nationalen Fachgesellschaften entwickelt und eingeführt, um zu überprüfen, ob Kollegen in Weiter-/Ausbildung über das fachspezifische Wissen verfügen, das von

2016

2017

2018

Alte Prüfungsform

Neue Prüfungsform

-

2016

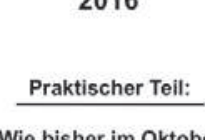

Wie bisher im Oktober

Schriftlicher Teil:

Wie bisher im Oktober

\begin{tabular}{c|}
2017 \\
$\frac{\text { Praktischer Teil: }}{\text { Wie bisher im Oktober }}$ \\
Schriftlicher Teil: \\
EEGC fakultativ im Juni \\
$\begin{array}{l}\text { Nach alter Prüfungsform } \\
\text { noch möglich im Oktober }\end{array}$
\end{tabular}

2018

Praktischer Teil:

Wie bisher im Oktober

Schriftlicher Teil:

EEGC obligatorisch im Juni

Nach alter Prüfungsform nicht mehr möglich einem frisch qualifizierten Facharzt* für Kardiologie erwartet wird. Dies stimmt mit dem Ziel überein, das die SGK hinsichtlich der schriftlichen Facharztprüfung verfolgt.

3. Die Guidelines der ESC sind für den kardiologischen Alltag in der Schweiz massgebend und werden von der SGK unterstützt. Das EEGC orientiert sich ebenfalls an den Guidelines der ESC.

4. Das EEGC verfügt über einen Fragenpool, der in $\mathrm{Zu}$ sammenarbeit mit den nationalen Gesellschaften regelmässig evaluiert, erweitert und erneuert wird.

5. Das EEGC erlaubt neben der Überprüfung des nötigen Basiswissens zudem, den Wissensstand der Kandidaten aus der Schweiz im europäischen Quervergleich zu evaluieren.

6. Das EEGC ist im Hinblick auf die zunehmende Mobilität der europäischen Bevölkerung bereits jetzt nützlich und wird in der Zukunft noch an Bedeutung gewinnen (möglicherweise im Sinne eines «European Cardiologist»).

Im Gegensatz zum schriftlichen Teil wird der praktische Teil der Facharzt-Prüfung unverändert beibehalten. Die praktischen Fähigkeiten und Fertigkeiten können mit einem MC-Examen nicht geprüft und sollen daher weiterhin am Fallbeispiel und am Patienten getestet werden.

Der Wechsel von der alten zur neuen schriftlichen Prüfung wird folgendermassen ablaufen (Abbildung):

- Im Jahr 2016 wird die schriftliche Facharzt-Prüfung wie bisher durchgeführt. Die Teilnahme am EEGC ist für Kandidaten aus der Schweiz noch nicht möglich.

- Für das Jahr 2017 ist eine Übergangslösung vorgesehen. Sie besteht darin, dass das EEGC und die schriftliche Prüfung in der alten Form parallel durchgeführt werden. Die Kandidaten können am EEGC teilnehmen; dies ist von Seiten der SGK erwünscht, aber nicht obligatorisch. Falls ein Kandidat das EEGC, welches im Juni stattfindet, besteht, ist er zur praktischen Prüfung automatisch zugelassen. Falls er das EEGC nicht besteht, kann er im Oktober die 
schriftliche Prüfung in der alten Form absolvieren. Die praktische Prüfung findet wie bisher am Tag nach der schriftlichen Prüfung in unveränderter Form statt.

- Ab dem Jahr 2018 ist geplant, ausschliesslich das EEGC als schriftliche Prüfung zu nutzen. Dies bedeutet, dass die Kandidaten die schriftliche Prüfung im Juni absolvieren müssen, die praktische Prüfung aber wie bisher erst im Oktober. Neu gilt, dass die Kandidaten die schriftliche Prüfung bestanden haben müssen, damit sie zur praktischen Prüfung zugelassen werden können. In diesem Zusammenhang wird empfohlen, die schriftliche Prüfung bereits im 3. Jahr der fachspezifischen Ausbildung abzulegen, die praktische Prüfung dagegen erst gegen Ende der fachspezifischen Ausbildung.

Die folgenden Lehrmittel werden zur Vorbereitung des EEGC empfohlen:

- ESC Textbook of Cardiovascular Medicine

- Guidelines der ESC und der ACC/AHA

- Online Lehrmittel:

- ESC eLearning: http://www.escardio.org/

- ACC Cardiosource: http://www.acc.org/

- Education in Heart: http://heart.bmj.com/site/ about/education.xhtml

Prof. Dr. med. Felix C. Tanne

Stv. Klinikdirektor

Kardiologie

Leitender Arzt Echokardio-

graphie

Universitäres Herzzentrum

Zürich

Rämistrasse 100

CH-8091 Zürich

\section{Wichtige Informationen für Kandidaten,}

\section{die am EEGC 2017 teilnehmen}

- Anmeldung bis 15.10.2016 an info[at]swisscardio.ch

- ESCTextbook of Cardiovascular Medicine als Geschenk nach Anmeldung

der in der deutsch- oder der französischsprachigen Schweiz absolviert werden. Die Korrespondenz mit den Kandidaten läuft ausschliesslich über das Sekretariat der SGK; die ESC wird mit den Kandidaten nicht in direkten Kontakt treten. Die Gebühr für das EEGC ist in der Prüfungsgebühr, die von der SGK für die gesamte Facharzt-Prüfung erhoben wird, enthalten.

Die SGK begrüsst es, wenn möglichst viele Kandidaten bereits im Jahr 2017 am EEGC teilnehmen. Die Anmeldung für das EEGC 2017 muss bis 15. Oktober 2016 beim Sekretariat SGK eingetroffen sein. Das EEGC 2017 wird am 15. Juni 2017 stattfinden. Die Kandidaten, die sich für das EEGC 2017 anmelden, erhalten zur Examensvorbereitung je ein ESC Textbook of Cardiovascular Medicine von der SGK geschenkt.

Der Vorstand der SGK ist überzeugt, mit dieser Anpassung der Facharzt-Prüfung die Qualität der Prüfung zu verbessern und ausserdem zur vermehrten Interaktion des kardiologischen Nachwuchses mit den Aktivitäten der ESC beizutragen, insbesondere den Guidelines und den Weiterbildungsmöglichkeiten.

Bildnachweis

zVg von den Autoren 